\title{
Sleep disorders in chronic obstructive pulmonary disease
}

\section{Dipti Gothi}

Associate Professor Pulmonary Medicine, Department of Pulmonary Medicine, ESI-PGIMSR, Delhi, India.

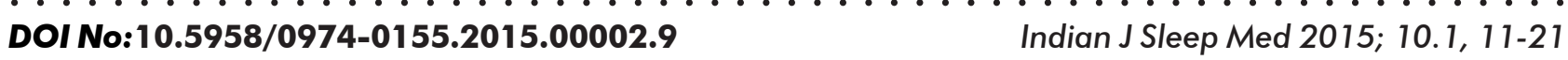

\begin{abstract}
Sleep disorders in chronic obstructive pulmonary disease (COPD) can occur in $60-70 \%$ of patients. They have adverse outcome in terms of both morbidity and mortality of COPD. The various sleep disorders seen in COPD are insomnia, restless leg syndrome (RLS), obstructive sleep apnea (OSA), nocturnal hypoventilation, and nocturnal oxygen desaturation (NOD). Of these disorders, insomnia and RLS can be diagnosed on history. OSA, sleep hypoventilation, and NODmay require type I polysomnography with/without transcutaneous or exhaled $\mathrm{CO}_{2}$ monitoring. Management consists of either conservative management or drug therapy for insomnia and RLS. If pharmacotherapy is required, ramelteon for insomnia and dopaminergic drug for RLS may be given. Treatment of OSA is continuous positive airway pressure, which relieves both OSA and COPD. Nocturnal hypoventilation usually requires oxygen along with noninvasive ventilator. Treatment of only NOD is controversial, but nocturnal oxygen may be given after ruling out nocturnal hypoventilation. This review emphasises the need of sleep disorders evaluation for comprehensive management of COPD.
\end{abstract}

Keywords:COPD, insomnia, restless leg syndrome, OSA, sleep hypoventilation, desaturation

\section{Introduction}

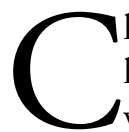

hronic obstructive pulmonary disease (COPD) has a high and increasing morbidity and mortality worldwide and is estimated to be the third leading cause of death by $2020^{1}$. COPD exhibit worse sleep quality and more sleep-related problems when compared with other chronic health problems ${ }^{2}$. Sleeprelated problems in COPD are insomnia,restless leg syndrome (RLS), overlap syndrome or comorbid obstructive sleep apnea (OSA), nocturnal sleep hypoventilation, and nocturnal oxygen desaturation $(\mathrm{NOD})^{3}$. These disorders diminish quality of life and lead to multifarious adverse consequences.

\section{Address for correspondence}

\section{Dr. Dipti Gothi, MD}

Associate Professor Pulmonary Medicine, Department of Pulmonary Medicine, ESI-PGIMSR, Delhi, India.

Email: diptigothi@gmail.com

\section{Prevalence}

More than $50 \%$ of patients report noteworthy disturbance in sleep quality ${ }^{4}$. It has been estimated that the prevalence of sleep disturbance varies from $34 \%$ to $78 \%$ in various studies $^{5-13}$. A study by Price et $\mathrm{al}^{13}$. showed that, although $78 \%$ of patients reported nighttime symptoms, only $67 \%$ of clinicians reported that their patients were bothered at night. Thus, the prevalence is underestimated. Although comprehensive evaluation of all sleep disorders in COPD has not been reported in a single study, different studies have evaluated different sleep disorders and shown prevalence as follows: RLS,29-36\% patients $^{14-16}$; overlap syndrome or comorbid OSA, 11-16\% patients $^{17,18}$; insomnia, $27-53 \%$ patients ${ }^{5,19}$; and NOD, $38-40 \%$ patients with moderate-to-severe COPD. Although the exact prevalence of sleep hypoventilation is not known in COPD, high body mass index (BMI), elevated baseline $\mathrm{PaCO}_{2}$, and increased time spent in rapid eye movement (REM) sleep are predictors of the severity of sleep hypoventilation ${ }^{3,20,21}$. 


\section{Pathogenesis}

The pathogenesis of sleep disorders is spearheaded by both sleep impacting respiration in COPD patients and impaction of COPD on sleep.

\section{Impact of Sleep on COPD,i.e.,Effect of Sleep on Ventilation in COPD Patients}

There are normal physiological adaptations during sleep, which result in mild hypoventilation in normal subjects. These changes are more pronounced during REM sleep compared with non-REM sleep ${ }^{22}$. These physiological changes during sleep are: (1) diminished respiratory center control in terms of decreased input and output resulting in reduced tidal volume and minute ventilation, ${ }^{23,24}(2)$ circadian changes in airway caliber leading to mild nocturnal bronchoconstriction, ${ }^{25}$ (3) decreased ribcage contribution to breathing owing to a marked reduction in accessory muscle activity, ${ }^{26}$ and (4) reduction in functional residual capacity (FRC) because of respiratory muscle hypotonia, cephalad displacement of the diaphragm, and a decrease in lung compliance leading to ventilation/perfusion (V/Q) mismatch. These changes are worse in COPD patients compared withnormal individuals ${ }^{22}$.

Apart from these physiological anomalies during sleep, COPD patients can also exhibit: (1) shift in the oxyhemoglobin dissociation curve owingto baseline hypoxemia. This leads to a greater fall in $\mathrm{SaO}_{2}$ when they hypoventilate during sleep ${ }^{1,17}$. (2) Accessary muscles activity, which is essential for some of the COPD patients, is compromised during sleep. This leads to massive reduction inFRC during sleep.

\section{Impact of COPD on Sleep}

Sleep is essential in humans to maintain cognitive performance and work productivity and physical, psychological, andemotional well-being. Sleep disturbances in COPD patients are common ${ }^{27}$. These sleep disturbances are related to demographic factors, such as age (most patients with COPD are older than 50 years of age), presence of obesity, pharmacotherapy, and disease-specific symptoms such as wheezing and $\operatorname{cough}^{28}$. Apart from these physiological and pathological aspects of COPD leading to sleep disturbances, they may also experience depression and RLS, which can compromise sleep. Thus, COPD also affects the sleep adversely.

Indian Journal of Sleep Medicine (IJSM), Vol. 10, No. 1, 2015

\section{Sleep Disorders in COPD Patients}

The common COPD and sleep-related disorders are insomnia, RLS, overlap syndrome, sleep hypoventilation, and NOD.

\section{Insomnia}

Definition: Insomnia is defined as difficulty falling asleep, staying asleep, waking up too early, or having unrefreshing sleep despite adequate opportunity and circumstances to sleep and it results in some form of daytime impairment ${ }^{3,29}$. Insomnia usually refers to chronic insomnia, which is present for at least a month unless otherwise stated ${ }^{30}$.

Prevalence: Insomnia prevalence varies from $27 \%$ to $53 \%{ }^{5,19}$ in various studies. The variation in the prevalence is owing to the variation in population and diagnostic methodology. Although the insomnia prevalence varies in various studies, it is definitely high in patients with COPD when compared with those without $\mathrm{COPD}^{31}$. Some studies suggest worse sleep in severe COPD, ${ }^{32}$ while other studies have not shown any association between forced expiratory volume in first second $\left(\mathrm{FEV}_{1}\right)$ and sleep quality ${ }^{17,33}$. Some studies have demonstrated a beneficial effect of supplemental oxygen, ${ }^{33-35}$ while others have not ${ }^{31,36}$. Presence of depression, anxiety, and current tobacco use, however, have shown consistently higher prevalence of insomnia in $\mathrm{COPD}^{3,33}$.

Etiology: Many factors are likely to be responsible for insomnia. These are ${ }^{3,33}$ : (a) respiratory symptoms including cough, sputum production, and dyspnea;(b) nicotine use, (c) nicotine withdrawal, (d) increased work of breathing, (e) hypoxia, (f) increased sympathetic activity, (g) comorbid anxiety and depression, (h) comorbid sleep disorders including OSA and RLS, and (i) use of medications such as theophylline. The exact role of each one leading to insomnia has not been ascertained. However, it appears that multiple factors may be responsible. Nocturnal hypoxia has been shown to cause increased sympathetic activity and hyperarousal ${ }^{37-38}$. However, studies have not shown improvement in insomnia with nocturnal oxygen therapy consistently. Thus, interplay of multiple factors with presence of underlying anxiety/depression seems to be responsible for insomnia ${ }^{39-40}$.

Consequences: Insomnia in COPD is associated with poor sleep quality ${ }^{18}$. The consequences of which are 
usually similar to that seen in general population. These consequences are increased risk of diabetes and hypertension leading to atherosclerosis, increased risk of myocardial infarction, and worse survival. They may also suffer from neurocognitive deficit such as poor concentration, memory, and irritability ${ }^{3}$.

Diagnosis: Insomnia is primarily a clinical diagnosis. ${ }^{3}$ A detailed inquiry regarding nocturnal and daytime symptoms and underlying precipitating factor should be made. Questionnaires are useful in determining the underlying cause, comorbid conditions, and the severity of daytime and nocturnal symptoms. There is a possibility of comorbid disease such as RLS, sleep apnea, nocturnal hypoxemia, and depression in patients of COPD. Global Sleep Assessment Questionnaire (GSAQ) is useful in ruling out some of these comorbid sleep disorder ${ }^{33}$. Epworth sleepiness score and fatigue severity index are useful in assessing daytime symptoms. Insomnia Severity Index is the most commonly used index for assessing the patient's perception of insomnia ${ }^{30}$. Pittsburgh Sleep Quality Index is yet another common index useful for measuring the quality of sleep ${ }^{30}$. When in doubt, a 2week sleep log should be completed before treatment. The sleep log should include bedtime, sleep latency, number of awakenings and duration of each awakening, wake after sleep onset, time in bed, total sleep time, sleep efficiency percentage,i.e., total sleep time/time in bed $\times 100$, and nap times ${ }^{30}$. Investigations such as actigraphy is largely limited to research arena whereas polysomnography (PSG) is useful in ruling out any other cause for poor quality ${ }^{3}$. Finally, an effort should be made to identify the cause of insomnia from among the underlying factors mentioned earlier.

Management: Evaluation for nocturnal oxygen therapy and RLS with smoking cessation and optimum management of COPD are the most important aspects of management of insomnia because of COPD. Cognitive behavioral therapy for insomnia (CBT-I) is recommended if insomnia persists after dealing with the above mentioned aspects of COPD. CBT-I is effective therapy in primary insomnia owing to COPD and appears to be superior to sedatives in the long-term, especially because of potential hazardous effect of pharmacotherapy ${ }^{41,42}$. Respiratory center depression and other adverse effects precludes the use of benzodiazepine in the management of insomnia owing to COPD. Instead, melatonin receptor agonist, i.e., ramelteon is preferred for the management of insomnia in COPD. Ramelteon (8mg per night) increases sleep efficiency without causing respiratory depression or worse hypoxemia ${ }^{42-43}$. Another drug, which may be helpful in the management, is doxepin, a histamine- 1 receptor antagonist. Low-dose (3-6 mg) doxepin is useful and less likely to cause anticholinergic side effect. Other histamine-1 receptor antagonists such astrazodone and mirtazapine have not been adequately tried ${ }^{3}$.

\section{Restless Leg Syndrome}

Definition: RLS is a somatosensory network disorder. Patients with RLS exhibit an urge to move and, generally, but not solely, affects the legs ${ }^{44,45}$.

Prevalence: Studies have reported that $29.1 \%$ to $36.8 \%$ of the COPD patients reveal RLS ${ }^{46}$. RLS is present in $2 \%$ to $15 \%$ of the general population ${ }^{47,48}$. The prevalence of RLS has been shown to be higher in COPD patients even in comparative studies between those with COPD and those without $\mathrm{COPD}^{49}$.

Etiology: Although the exact pathogenesis of RLS is not known, clinical observations suggest the role of dopaminergic pathway as dopaminergic drugs that alleviate the symptoms and dopamine antagonists trigger or exacerbate them ${ }^{50}$. The impairment in dopaminergic system is thought to be functional and metabolic rather than anatomical. The exact mechanism by which RLS affects COPD is yet to be clearly elucidated. However, hypoxemia and/or hypercapnia have been considered as causative mechanism for RLS. Hypoxia, through the hypoxia-inducible factor-1 pathway, may lead to an increase in tyrosine hydroxylase and vascular endothelial growth factor. The former is a rate-limiting enzyme in dopamine synthesis and is increased in RLS leading to depletion of dopamine ${ }^{3}$.

Consequences: RLS exerts a huge impact on quality of sleep and quality of life in general ${ }^{3}$. Owing to poor sleep quality, RLS patients are more prone to the risk of depression, anxiety, and panic disorder ${ }^{51}$. Presence of COPD can have an additive effect on neuropsychiatric consequences of RLS. Evolving evidence also proposes that RLS is accompanied with metabolic dysregulation and autonomic dysfunction. It has been shown that RLS is probably associated with diabetes and impaired glucose tolerance ${ }^{52}$.

Diagnosis: Diagnosis of RLS is based on a typical history and does not need a PSG for confirmation. However, RLS can be secondary to multiple causes ranging from anemia, cardiovascular disease, obesity, diabetes, 
rheumatological disorders peripheral neuropathy, to neurological disorders. These secondary causes should be ruled out before labeling it as RLS owingto COPD.

There are four essential diagnostic criteria first defined in 1995 and subsequently revised in $2003^{53,54}$. The diagnosis does not require any laboratory test, but it is essential to fulfill all the criteria:

- An urge to move the legs, usually associated with unpleasant leg sensations

- Exacerbation of symptoms by rest

- Symptom relief on activity

- Symptoms worse in the evening and at night.

The pneumonic for the same can be "URGE" 55 : urge to move, rest induced, gets better with activity, and evening and night accentuation.

Management: No specific studies have been conducted to assess the therapy of RLS specifically in COPD patients. For patients with mild or infrequent symptoms, nondrug-based options may be sufficient to provide symptom relief. It has been observed that only about $20 \%$ RLS patients require drugs ${ }^{56}$. The nonpharmacological management consists of avoidance of alcohol, caffeine, smoking, over exertion, stress and sleep deprivation, and following good sleep hygiene, regular exercise, and relaxation techniques.

Pharmacological treatment is only required in patients with $^{50,57}$ symptoms that seriously impair quality of life, sleep, or daytime functioning and in whom there are more than two attacks per week despite reversal of iron deficiency and removal of possible exacerbators/ secondary cause ${ }^{45}$. The pharmacological treatment primarily consists of dopaminergic agents such asropinirole, pramipexole, and rotigotine; $\alpha 2$ calciumchannel ligands such as gabapentin and pregabalin; opioid agonist such as tramadol and methadone; and benzodiazepines $^{57}$. Of these, ropinirole, $1.6-2.0 \mathrm{mg}$; pramipexole (immediate/extended release), 0.125$0.75 \mathrm{mg} /$ day; and rotigotine (patch), $1-3 \mathrm{mg}$, are the most extensively investigated and effective therapies for the treatment of RLS and generally considered the first-line agents. Augmentation and impulse control disorder (ICD) are the commonly observed side effects with these drugs. In case of side effects, it is recommended that $\alpha 2$ calcium-channel ligands, which are the second most commonly investigated drugs, should be used. Somnolence and dizziness are the commonly observed side effects with $\alpha 2$ calcium-channel ligands such as pregabalin $(100-300 \mathrm{mg} /$ day) and gabapentin $(300 \mathrm{mg} /$ day) ${ }^{58}$. The other drugs are used only in refractory cases as they are less effective, exertmore side effects, or have not been well investigated.

\section{Overlap Syndrome}

Definition: Concurrence of OSA and COPD is termed as "overlap syndrome $e^{3}$."

Prevalence: The prevalence of overlap syndrome is very different depending on the definition used in various studies. However, one largest study of 5,954 participants, done in conjunction with the Sleep Heart Health Study, showed prevalence of OSA $14.0 \%$ among COPD subjects and $18.6 \%$ in those without $\mathrm{COPD}^{59}$. Thus, there is no difference between the prevalence of OSA in general population and that in COPD patients.

Clinical features: The most common symptoms of OSA patients include chronic loud snoring, excessive daytime sleepiness (EDS), personality changes, and deterioration of quality of life. Compared with the OSA-only group, the overlap population tends to be older with higher $\mathrm{BMI}^{59}$. The BMI is usually higher than in patients with COPD alone but lower than in patients with only OSA. They commonly do not show EDS, which makes diagnosis difficult ${ }^{60}$. They commonly reveal hypoxemia and hypercapnia and higher mean pulmonary artery pressures $^{61}$.

Etiology: Althoughthe coexistence of COPD and OSA may likely just be a chance occurrence, some of the studies have found the co-occurrence of the two disorders more than mere coexistence ${ }^{62}$. Thus, there is a possibility of one predisposing the other. This possibility of increased prevalence of OSA in COPD and vice versa is possibly owing to aggravated physiological changes during sleep as mentioned earlier in patients with COPD, which may lead to easy upper airway collapsibility ${ }^{3}$. In addition, COPD patients may be using high doses of steroid, which can cause increased neck size and myopathy. Moreover, because of increased upper airway edema from cor pulmonale, COPD patients may be at increased risk of developing upper airway obstruction $^{3}$.

There is also a possibility of OSA aggravating COPD because OSA leads to systemic inflammation, which may contribute to lower airway inflammation ${ }^{3}$. Hypoxia from OSA can cause upregulation of xanthine oxide reductase 
in pulmonary endothelial cells $s^{63}$, similar to that produced by tobacco smoke $^{64}$, which could also contribute to the pathogenesis of COPD. Finally, smoking, obesity, allergic rhinitis, and gastroesophageal reflux disease (GERD) if present in either of the disorders can predispose to the risk having the other disorder ${ }^{3}$.

Consequences: Far worse than the possibility of two disorders coexisting together is the consequences of having both together. Complications of overlap syndrome are high because overlap syndrome patients show higher risk of prolonged oxygen desaturation at night than those with OSA alone as both COPD and OSA can lead to hypoxemia during sleep ${ }^{60}$. Prolonged hypoxemia increases the morbidity and mortality considerably ${ }^{59}$. It predisposes to daytime hypercapnia and hypoxemia, which is independent of lung function ${ }^{65}$. Pulmonary hypertension is also higher in OSA (16\%) compared with overlap syndrome $(86 \%)^{66}$.

Both COPD and OSA are known to cause systemic inflammation and increased cardiovascular risk ${ }^{67}$. Thus, there are likelihood of increased prevalence of cardiovascular complications among patients with overlap $^{68}$. Comorbid OSA has been reported to have increase mortality in patients with COPD by Marin et $\mathrm{a}^{69}$. The study involved over 600 patients exhibiting COPD alone and overlap syndrome with and without continuous positive airway pressure (CPAP) treatment. It was shown that there was significantly higher mortality and number of cardiovascular deaths in patients with untreated overlap syndrome compared with treated overlap syndrome patients and only COPD patients. Moreover, the morbidity, i.e., exacerbations owing to COPD, was also significantly higher in overlap group compared with the other two groups ${ }^{69}$. Recent work by Mermigkis et $\mathrm{al}^{70}$. showed that, in addition to increased morbidity and mortality, patients with the overlap syndrome also experience significantly worse quality of life measured with the St George's Respiratory Questionnaire, when compared with COPD-only control subjects $^{70}$. So, overlap syndrome increases mortality and morbidity and decreases quality of life, which can be significantly improved with CPAP therapy ${ }^{68}$.

Diagnosis: COPD patients with symptoms suggestive of OSA, such as snoring or witnessed apneas, ${ }^{3}$ should be evaluated by PSG, as EDS is not common in patients with overlap syndrome ${ }^{61}$. In addition, in patients with COPD, presence of pulmonary hypertension out of proportion to the disease severity may indicate comorbid
OSA, and PSG should be considered ${ }^{71}$. The diagnosis of OSA is based on clinical findings along with a full-night PSG showing apnea-hypopnea index $(\mathrm{AHI})>5$. Type I studies,i.e., in-laboratory PSG with neurocardiorespiratory channels, are preferred over type III studies (limited or cardiorespiratory PCSG). This is because type III studies are not validated in overlap syndrome, ${ }^{72}$ $\mathrm{CO}_{2}$ monitoring for hypoventilation may be required, and accompanying insomniacan be ruled out. Similarly, in-laboratory titrationPSG may be better than auto-CPAP titration.

Management: Management of comorbidities such as allergic rhinitis and GERD is important. Similarly, smoking cessation, lifestyle changes, and obesity management also have salutary effect on both OSA and $\mathrm{COPD}^{3}$. CPAP does remain the standard of care for overlap syndrome such as OSA ${ }^{59}$. In fact, CPAP not only improves AHI but also counteracts auto-PEEP generated owing to COPD. CPAP also exerta mild bronchodilator effect, because overall inflammatory stimulus to airways is reduced ${ }^{73}$. So, CPAP works as double-edged sword for the management of overlap syndrome. Correspondingly, the trial have shown that CPAP helps in improvement of sleep apnea symptoms, spirometric parameters ( $\mathrm{FEV}_{1}$ and forced vital capacity), and gas exchange $\left(\mathrm{PaO}_{2} \text { and } \mathrm{PaCO}_{2}\right)^{73}$. CPAP also reduces the number of COPD-related severe exacerbations and hospital admissions ${ }^{74}$. In a prospective cohort of 95 patients with moderate/severe COPD (GOLD stages IIIII) and moderate/severe OSA (AHI > 15), 5-year survival estimate was $71 \%$ in CPAP-treated patients compared with $26 \%$ in patients on long-term oxygen therapy (LTOT) alone ${ }^{75} .{ }^{75}$ Similarly, in a trial by Marin et al ${ }^{69}$. described earlier, it was shown that overlap syndrome patients treated with CPAP revealed significant survival benefit over those not treated with $\mathrm{CPAP}^{69}$. It is noteworthy that oxygen therapy, which is useful in COPD-induced hypoxemia, shows a detrimental effect on overlap syndrome. This is because oxygen therapy worsens $\mathrm{CO}_{2}$ retention ${ }^{3}$. NIPPV and Bi-PAP have not been adequately tried in overlap syndrome, but it may have beneficiary effect in patients with associated hypoventilation ${ }^{76}$. There are no studies on oral appliances in overlap syndrome ${ }^{3}$.

\section{Sleep Hypoventilation}

Definition: American Academy of Sleep Medicine (AASM) in 2012 has revised the definition of nocturnal hypoventilation to " $\mathrm{An}$ increase in the $\mathrm{PaCO}_{2}$ to $>55$ $\mathrm{mm} \mathrm{Hg}$ for $\geq 10$ minutes, or an increase in the $\mathrm{PaCO}_{2}$ by

Indian Journal of Sleep Medicine (IJSM), Vol. 10, No. 1, 2015 
$\geq 10 \mathrm{~mm} \mathrm{Hg}$ above the awake supine value to a value over $50 \mathrm{~mm} \mathrm{Hg}$ for $\geq 10$ minutes" ${ }^{\prime \prime 7}$. The recent AASM guidelines have also suggested that end-tidal $\mathrm{PCO}_{2}$ $\left(\mathrm{PETCO}_{2}\right)$ or transcutaneous $\mathrm{PCO}_{2}\left(\mathrm{tcPCO}_{2}\right)$ may be used as surrogates of arterial $\mathrm{PaCO}_{2}$ for diagnostic and $\mathrm{tcPCO}_{2}$ for titration PSG. Since the guidelines have included $\mathrm{PETCO}_{2}$ and $\mathrm{tcCO}_{2}$, it is much easier to detect hypoventilation now than it was before the recent definition.

Prevalence: Owing to difficulty in monitoring $\mathrm{PaCO}_{2}$ during sleep, data regarding sleep-related hypoventilation in COPD are scarce. In one of the studies on 54 stable hypercapnic COPD patients without concomitant sleep apnea or morbid obesity, $43 \%$ were found to reveal sleep hypoventilation ${ }^{78}$. In yet another study of 80 clinically stable hypercapnic COPD patients, $21 \%$ developed nocturnal hypoventilation after a night of supplemental oxygen therapy ${ }^{79}$. It is important to note that sleep hypoventilation can develop on receiving oxygen therapy which is an integral part of COPD management ${ }^{80}$. Although most of the studies available are on stable hypercapnic patients, it is not essential that patients of sleep hypoventilation always exhibit daytime hypercapnia. Sleep hypoventilation can also occur in normocapnic, non-LTOT subjects ${ }^{81}$.

Clinical features: COPD with sleep hypoventilation patients commonly experience morning headaches, restless sleep, daytime sleepiness, orthopnea, recurrent respiratory problems, and corpulmonale ${ }^{80}$.

Etiology: The mean $\mathrm{PtcCO}_{2}$ increases with sleep stages and greater between deeper stages of sleep in subjects with sleep hypoventilation versus those without sleep hypoventilation. This supports the notion that, in COPD, sleep hypoventilation is primarily the consequence of a failing respiratory pump ${ }^{81}$. As arterial $\mathrm{CO}_{2}$ increases owing to hypoventilation, the brains' extracellular fluid bicarbonate builds up, blunting the central ventilatory drive, which in turn leads to further elevation of $\mathrm{CO}_{2}^{82}$. Thus, patients with daytime hypercapnia are more likely to have nocturnal hypoventilation owing to their elevated baseline bicarbonate levels. But, apart from daytime hypercapnia, diminished ventilatory drive, increased upper airway resistance, and mechanical disadvantages imposed by hyperinflation also contribute toward nocturnal hypoventilation ${ }^{3}$. Studies have also shown that increased BMI contributes to the severity of sleep hypoventilation ${ }^{3}$.
None of the available studies have shown direct correlation of sleep hypoventilation with poor lung function in COPD. In one of the studies, it was shown that COPD patients with mean $\mathrm{FEV}_{1}$ of $1.0 \mathrm{Land} \mathrm{PaO}_{2}$ ranging from 56 to $69 \mathrm{~mm} \mathrm{Hg}$ revealed a high likelihood of developing nocturnal desaturation ${ }^{52}$. This study was performed before the revised definition of hypoventilation; hence, nocturnal $\mathrm{CO}_{2}$ monitoring was not performed. It is possible that some of these patients experienced nocturnal hypoventilation. Thus, it is possible that those with poor lung function show more likelihood of developing nocturnal hypoventilation.

Consequences: Daytime hypercapnia can be a both cause and consequence of sleep hypoventilation. Irrespective of whether it is cause or consequence, hypercapnia is known to decrease myocardial and diaphragmatic contractility, increase pulmonary artery pressure, and predispose to arrhythmias. Some of the consequences of hypoventilation, such as cor pulmonale and pulmonary hypertension, may be irreversible ${ }^{83}$. Sleep-related hypoventilation is also associated with a reduced life expectancy ${ }^{84}$.

Diagnosis: Morning headaches in patients with COPD may suggest nocturnal hypoventilation. If nocturnal $\mathrm{CO}_{2}$ retention is suspected, $\mathrm{CO}_{2}$ monitoring should be considered along with the PSG ${ }^{3}$. Sleep hypoventilation can be easily missed and lead to detrimental outcome. Hence, overnight screening with pulse oximetry is important, especially before prescribing LTOT. If it shows an evidence of nocturnal desaturation, an overnight PSG with $\mathrm{PETCO}_{2}$ or $\mathrm{tcCO}_{2}$ or intraarterial monitoring should be done. Since poor lung function $\left(\mathrm{FEV}_{1}<49 \%,<1 \mathrm{~L}\right)$ is a predisposing factor for nocturnal desaturation and daytime hypercapna and obesity have been definitely correlated with hypoventilation, the overnight screening is indicated in these cases as well ${ }^{52,85}$. The reliability and validity of these surrogates, especially in severe COPD, may be limited, and correlation with arterial blood gas analysis should be performed before performing the study ${ }^{3}$.

Treatment: Noninvasive ventilation (NIV) during sleep ameliorates nocturnal hypoventilation, leading to a downward resetting of the respiratory center sensitivity for $\mathrm{CO}_{2}$ and improvement in nocturnal and daytime $\mathrm{PaCO}_{2}{ }^{26}$. Daytime improvement in gas exchange leads to improved sleep quality, daytime function, and quality of life. Some of the studies have also shown survival benefit ${ }^{87}$. The earlier studies performed on patients with 
chronic respiratory failure have shown that the average baseline $\mathrm{PaCO}_{2}$ improves better if they have higher daytime $\mathrm{PaCO}_{2}{ }^{87}$. Studies specifically evaluating nocturnal hypoventilation and its effect on quality of life and survival benefit have not been performed. Thus, the guidelines accordingly for prescription of NIV laid down in 1999 and reiterated in 2006 are $^{87,88}$ : (1) symptomatic patient where OSA is excluded and (2) daytime $\mathrm{PaCO}_{2}$ is $>55$ $\mathrm{mmHg}$ or (3) daytime $\mathrm{PaCO}_{2}$ is $50-54 \mathrm{mmHg}$ with either evidence of nocturnal hypoventilation based on nocturnal oximetry showing sustained desaturation to $<89 \%$ for $>5$ min while patient is on his or her usual $\mathrm{FiO}_{2}$ or repeated hospitalizations ( $>2$ in a 12 -month period). Because guidelines specifically for management of nocturnal hypoventilation are not available, these guidelines are followed for nocturnal hypoventilation as well. Studies are required for patients with only nocturnal hypoventilation and its correlation with survival and quality of life benefit with NIV.

Average volume assured pressure support (AVAPS; Philips Respironics) and intelligent volume assured pressure support (iVAPS; ResMed) are newer hybrid modes that use proprietary algorithms to calculate the pressure support needed to achieve a target tidal volume or alveolar ventilation, respectively. In stable chronic hypercapnic COPD patients, iVAPS showed a greater decrease in nocturnal hypercapnia and a trend toward more restful sleep at 6 weeks of treatment ${ }^{89}$. Studies are required to show its benefit in sleep hypoventilation ${ }^{3}$.

\section{Nocturnal Oxygen Desaturation}

Definition: There is no formal definition of NOD, and various studies use different definitions. As per the available studies/literature, it is given as "Oxygen saturation during sleep to $<90 \%$ for more than five minutes with a nadir of at least $85 \%$ or $>30 \%$ of total sleep time with $\mathrm{SpO}_{2}$ of $<90 \%{ }^{\prime 90-92}$. It should be noted that nocturnal hypoventilation can also give rise to similar desaturation at night. Nocturnal $\mathrm{CO}_{2}$ monitoring can only distinguish the two. Presence of only NOD is owing to lung failure and the nocturnal hypoventilation is owing to pump failure. The consequences of both can be different. The management of NOD also differs from nocturnal hypoventilation. Thus, an effort should be made to exclude sleep hypoventilation before diagnosing NOD.

Prevalence: NOD has been reported in $40-70 \%$ of COPD patients with daytime saturations between $90 \%$ and $95 \% 85,90,92$. It is also estimated that patients with $\mathrm{FEV}_{1}>1.45 \mathrm{~L}$ or $>49 \%$ predicted usually do not experience nocturnal desaturation ${ }^{85}$. There have been no studies on NOD, which have separated hypoventilation from nocturnal hypoxemia. Thus, the prevalence of only NOD without hypoventilation is not known.

Etiology: Alveolar hypoventilation leading to decreased minute ventilation may be the primary mechanism of nocturnal hypoxemia. Minute ventilation can drop approximately by $16 \%$ during non-REM sleep and 32\% during REM sleep in patients with COPD ${ }^{93}$. This is because, during wakefulness, respiration is not only under metabolic control but also influenced by voluntary processes such as speaking and swallowing, and during sleep, chemoreceptors and ventilatory centers are the sole controllers of respiration. This leads to drop in $\mathrm{PaO}_{2}$ by $3-10 \mathrm{~mm} \mathrm{Hg}$ and elevation in $\mathrm{PaCO}_{2}$ by $2-8 \mathrm{~mm} \mathrm{Hg}$ during sleep. Those who have blunted receptors for $\mathrm{CO}_{2}$ may develop sleep hypoventilation, whereas those with high chemosensitivity to $\mathrm{CO}_{2}$ may develop NOD. Patients with borderline saturation of $93 \%$ or below may have saturation falling into steep part of oxyhemoglob in dissociation curve at night leading to significant drop in $\mathrm{PaO}_{2}$. Hence, daytime oxygen saturation is among the strongest predictors of nocturnal desaturation in patients with $\mathrm{COPD}^{94}$.

Consequences: Acute episodes of nocturnal desaturation can cause elevation in systemic systolic and mean pulmonary artery blood pressures ${ }^{95}$. These repetitive and transient desaturations overtime can lead to chronic pulmonary hypertension. Systematic inflammation is positively activated by $\mathrm{NOD}^{96}$. Sergi et $\mathrm{a}^{97}$. found that NOD in COPD patients may represent an independent risk factor for the development of chronic respiratory failure in COPD patients with daytime $\mathrm{PaO}_{2}>60 \mathrm{~mm}$ $\mathrm{Hg}$. Cardiac arrhythmias have also been linked with nocturnal desaturations ${ }^{98}$ and may contribute to the higher than expected nocturnal death rate in COPD patients ${ }^{99}$. In a retrospective study of 169 patients with COPD, Fletcher et $\mathrm{al}^{100}$. noted significantly decreased survival among patients with NOD compared with those without. It needs to be noted that these studies have been based on only oxygenmonitoring and not with $\mathrm{CO}_{2}$ monitoring. Hence, only hypoxia or both hypoxia and hypercarbia are the culprits is not known.

Diagnosis: COPD patients with relatively low daytime saturation $(<93 \%)$ and moderate-to-severe COPD may be considered for overnight oximetry ${ }^{3,85}$. Home oximetry

Indian Journal of Sleep Medicine (IJSM), Vol. 10, No. 1, 2015 
is an effective practical method for screening this population ${ }^{19}$. A cyclical (sawtooth) pattern on overnight oximetry suggests sleep-disordered breathing ${ }^{19}$. If screening shows an evidence of NOD, PSG with $\mathrm{CO}_{2}$ monitoring should be performed.

Management: The necessity of treatment of isolated NOD in COPD has been debated for many years ${ }^{101}$. This is because: (1) To date, only two randomized trials have addressed the issue of nocturnal oxygen therapy in patients with COPD with significant NOD who would not qualify for LTOT ${ }^{102,103}$. Meta-analysis of which did not show survival benefit (pooled odds ratio: 0.97; 95\% CI: 0.41-2.31) ${ }^{104}$ However, since the number of patients in these trials was small, nothing can be concluded ${ }^{19}$. (2) There has been no systemic study, which has separated NOD from nocturnal hypoventilation in daytime normocapnic, non-LTOT patients and evaluated the use of nocturnal oxygen versus NIV. Nevertheless, Medicare guidelines allow the use of nocturnal oxygen in daytime normoxemic patients based on indirect comparison of the British Medical Research Council Study ${ }^{104}$ and the National Heart Lung and Blood Institute's trial ${ }^{104}$ showing that patients receiving $12 \mathrm{~h}$ of oxygen/day (most of which was provided at night) show better survival than those receiving no oxygen therapy ${ }^{19}$. However, more research is required for determining use of nocturnal oxygen therapy. Among other modalities of treatment for NOD, optimum treatment with bronchodilator and lung volume reduction surgery decreases airflow obstruction, airtrapping, and hyperinflation and improves sleep quality and nocturnal oxygenation ${ }^{105}$.

\section{Conclusion}

Sleep is often a forgotten dimension in patients with COPD. COPD patients show worse sleep quality and more sleep-related problems when compared with patients with other chronic health problems. Some of the sleep disorders in COPD such as insomnia and RLS can easily be diagnosed and treated. The others such as OSA can exert a huge impact on morbidity and mortality and can be easily managed with CPAP. Nocturnal hypoventilation and NOD are poorly studied; hence, more studies are required for these two disorders. Nevertheless, both can lead to adversity in terms of morbidity and mortality. Thus, sleep, which is an important aspect of life, should not be forgotten while treating the third leading cause of death of the century.

\section{References}

1. Lopez AD, Shibuya K, Rao C, Mathers CD, Hansell AL, Held LS, et al. Chronic obstructive pulmonary disease: current burden and future projections. Eur Respir J. 2006;27:397-412.

2. Ezzie ME, Parsons JP, Mastronarde JG. Sleep and obstructive lung diseases. Sleep Med Clin. 2008;3:505-515.

3. Budhiraja R, Siddiqi TA, Quan SF. Sleep disorders inchronic obstructive pulmonary disease: etiology, impact, and management. J Clin Sleep Med. 2015;11:259-270.

4. Sleep quality in COPD. Available at:http:// www.thoracic.org/copd-guidelines/for-healthprofessionals/management-of-stable-copd/sleep/sleepproblems-in-copd.php(accessed on September 21, 2012).

5. Klink M, Quan SF. Prevalence of reported sleep disturbances in a general adult population and their relationship to obstructive airways diseases. Chest. 1987;91:540-546.

6. Klink ME, Dodge R, Quan SF. The relation of sleep complaints to respiratory symptoms in a general population. Chest. 1994;105:151-154.

7. Lewis CA, Fergusson W, Eaton T, Zeng I, Kolbe. Isolated nocturnal desaturation in COPD: prevalence and impact on quality of life and sleep. Thorax. 2009;64:133-138.

8. Cormick W, Olson LG, Hensley MJ, Saunders NA. Nocturnal hypoxaemia and quality of sleep in patients with chronic obstructive lung disease. Thorax. 1986;41:846-854.

9. Valipour A, Lavie P, Lothaller H, Mikulic I, Burghuber OC. Sleep profile and symptoms of sleep disorders in patients with stable mild to moderate chronic obstructive pulmonary disease. Sleep Med. $2011 ; 12: 367-372$.

10. Kinsman RA, Yaroush RA, Fernandez E, Dirks JF, Schocket $M$, Fukuhara J. Symptoms and experiences in chronic bronchitis and emphysema. Chest. 1983;83:755-761.

11. Anzueto A, Ferguson GT, Feldman G, et al. Effect of fluticasone propionate/salmeterol $(250 / 50)$ on COPD exacerbations and impact on patient outcomes. COPD. $2009 ; 6: 320-329$.

12. Tashkin DP, Rennard SI, Martin P, et al. Efficacy and safety of budesonide and formoterol in one pressurized metereddose inhaler in patients with moderate to very severe chronic obstructive pulmonary disease: results of a 6-month randomized clinical trial. Drugs. 2008;68:1975-2000.

13. Price D, Small M, Milligan G, Higgins V, Gil EG, Estruch J. The prevalence and impact of night-time symptoms in COPD-a real-world study in five European countries. Int $J$ Chron Obstruct Pulmon Dis.2013;8:595-603.

14. Kaplan $Y$, Inonu H,Yilmaz A, Ocal S.Restless legs syndrome in patients with chronic obstructive pulmonary disease.Can J Neurol Sci.2008;35:352-357.

15. Lo Coco D, Mattaliano A, Lo Coco A, Randisi B. Increased frequency of restless legs syndrome in chronic obstructive pulmonary disease patients. Sleep Med.2009;10:572-576.

16. Cavalcante AG, de Bruin PF, de Bruin VM, et al.Restless legs syndrome, sleep impairment, and fatigue in chronic obstructive pulmonary disease. Sleep Med. 2012;13: 842-847. 
17. Budhiraja R, Parthasarathy S, Budhiraja P, Habib MP, Wendel C, Quan SF. Insomnia in patients with COPD. Sleep. 2012;35:369-75.

18. Lee $\mathbf{R}, \mathrm{McNicholas}$ WT. Obstructive sleep apnea in chronic obstructive pulmonary disease patients. Curr Opin Pulm Med. $2011 ; 17: 79-83$.

19. Lacasse $Y$, Sériès $F$, Vujovic-Zotovic $N$, et al.Evaluating nocturnal oxygen desaturation in COPD revised. Respir Med.2011;105:1331-1337.

20. INOX Trial Group.Characteristics of patients with COPD and nocturnal oxygen desaturation. Eur Resp J. 2014;44(Suppl 58):P3698.

21. McNicholas WT. Impact of sleep in COPD. Chest.2000;117:48S-53S.

22. Douglas NJ, White DP, Pickett CK, Weil JV, Zwillich CW. Respiration during sleep in normal man. Thorax. 1982;37:840-844.

23. Krieger J, Turlot JC, Mangin P, Kurtz D. Breathing during sleep in normal young and elderly subjects: hypopneas, apneas andcorrelated factors. Sleep. 1983;6:108-120.

24. Kerr HD. Diurnal variation of respiratory function independent of air quality. Arch Environ Health. 1973;26:144-153.

25. Tusiewicz K, Moldofsky H, Bryan AC, Bryan MH. Mechanics of the ribcage and diaphragm during sleep. J Appl Physiol Respir Environ Excer Physiol. 1977;43:600-602.

26. Agusti A, Hedner J, Marin JM,Barbé F.Cazzola M,Rennard S.Night-time symptoms: a forgotten dimension of COPD. Eur Respir Rev. 2011;20:183-194.

27. Weitzenblum E, Chaouat A. Sleep and chronic obstructive pulmonary disease. Sleep Med Rev. 2004;8:281-294.

28. American Academy of Sleep Medicine. The International Classification of Sleep Disorders, 3rd ed. Darien, IL: American Academy of Sleep Medicine, 2014.

29. Schutte-Rodin S, Broch L, Buysse D, Dorsey C, Sateia M. Clinical guideline for the evaluation and management of chronic insomnia in adults. J Clin Sleep Med. 2008;4:487-504.

30. Budhiraja R, Roth T, Hudgel DW, Budhiraja P, Drake CL. Prevalence and polysomnographic correlates of insomnia comorbid with medical disorders. Sleep. $2011 ; 34: 859-867$.

31. Fleetham J, West P, Mezon B, Conway W, Roth T, Kryger M. Sleep, arousals, and oxygen desaturation in chronic obstructive pulmonary disease. The effect of oxygen therapy. Am Rev Respir Dis. 1982;126:429-433.

32. Omachi TA, Blanc PD, Claman DM, et al. Disturbed sleep among COPD patients is longitudinally associated with mortality and adverse COPD outcomes. Sleep Med. $2012 ; 13: 476-483$.

33. Calverley PM, Brezinova V, Douglas NJ, Catterall JR, Flenley DC. The effect of oxygenation on sleep quality in chronic bronchitis and emphysema. Am Rev Respir Dis. $1982 ; 126: 206-210$.

34. Goldstein RS, Ramcharan V, Bowes G, McNicholas WT, Bradley D, Phillipson EA. Effect of supplemental nocturnal oxygen on gas exchange in patients with severe obstructive lung disease. N Engl J Med. 1984;310:425-429.
35. McKeon JL, Murree-Allen K, Saunders NA. Supplemental oxygen and quality of sleep in patients with chronic obstructive lung disease. Thorax.1989;44:184-188.

36. Bonnet MH, Arand DL. Hyperarousal and insomnia: state of the science. Sleep Med Rev. 2010;14:9-15.

37. Sica AL, Greenberg HE, Ruggiero DA, Scharf SM. Chronicintermittent hypoxia: a model of sympathetic activation in the rat. Respir Physiol. 2000;121:173-184.

38. Schnell K, Weiss CO, Lee T, Krishnan JA, Leff B, Wolff JL, et al. The prevalence of clinically-relevant comorbid conditions in patients with physician-diagnosed COPD: a crosssectional study using data from NHANES 1999-2008. BMC Pulm Med. 2012;12:26.

39. Sundh J, Ställberg B, Lisspers K, Montgomery SM, Janson C. Co-morbidity, body mass index and quality of life in COPD using the Clinical COPD Questionnaire. COPD. 2011;8:173-181

40. Mitchell MD, Gehrman P, Perlis $M$, Umscheid CA Comparative effectiveness of cognitive behavioral therapy for insomnia: a systematic review. BMC Fam Pract. $2012 ; 13: 40$.

41. Morgenthaler T, Kramer M, Alessi C, et al. Practice parameters for the psychological and behavioral treatment of insomnia: an update. An American Academy of Sleep Medicine report. Sleep. 2006;29:1415-1419.

42. Kryger $\mathbf{M}$, Roth T, Wang-Weigand S, Zhang J. The effects of ramelteon on respiration during sleep in subjects with moderate to severe chronic obstructive pulmonary disease. Sleep Breath. 2009;13:79-84.

43. Nunes DM, Mota RM, Machado MO, Pereira ED, Bruin VM Bruin PF. Effect of melatonin administration on subjective sleep quality in chronic obstructive pulmonary disease. Braz J Med Biol Res. 2008;41:926-923.

44. GothiD. Restless legs syndrome: common yet an under recognized entity. Indian J Sleep Med. 2014;9:41-49.

45. Aras G, Kadakal F,Purisa S,Kanmaz D,Aynaci A,Isik E. Are we aware of restless legs syndrome in COPD patients who are in an exacerbation period? Frequency and probable factors related to underlying mechanism. COPD. $2011 ; 8: 437-443$

46. Allen RP, Walters AS, Montplaisir J, Hening W, Myers A, Bell $\mathrm{TJ}$, et al. Restless legs syndrome prevalence and impact: REST general population study. Arch Intern Med. 2005; 165:1286-1292.

47. Allen RP, Stillman P, Myers AJ. Physician-diagnosed restless legs syndrome in a large sample of primary medical care patients in western Europe: prevalence and characteristics. Sleep Med. 2010;11:31-37.

48. Nichols DA, Allen RP, Grauke JH, et al. Restless legs syndrome symptoms in primary care: a prevalence study. Arch Intern Med. 2003;163:2323-2329.

49. Leschziner G, Gringras P. Restless legs syndrome. BMJ. $2012 ; 344: \mathrm{e} 3056$.

50. Winkelman JW, Finn L, Young T. Prevalence and correlates of restless legssyndrome symptoms in the Wisconsin Sleep Cohort. Sleep Med. 2006;7:545-552.

Indian Journal of Sleep Medicine (IJSM), Vol. 10, No. 1, 2015 
51. Innes KE, Selfe TK, Agarwal P. Restless legs syndrome and conditions associated with metabolic dysregulation, sympathoadrenal dysfunction, and cardiovascular disease risk: a systematic review. Sleep Med Rev. 2012;16:309-339.

52. Walters AS. Toward a better definition of restless legs syndrome. The International Restless Legs Syndrome Study Group. Mov Disord. 1995;10:634-642.

53. Allen RP, Picchietti D, Hening WA, Trenkwalder C, Walters AS, Montplaisi J; Restless Legs Syndrome Diagnosis and Epidemiology workshop at the National Institutes of Health; International Restless Legs Syndrome Study Group.Restless legs syndrome: diagnostic criteria, special considerations, and epidemiology. A report from the restless legs syndrome diagnosis and epidemiology workshop at the National Institutes of Health. Sleep Med. 2003;4:101-119.

54. Hening W, Allen RP, Tenzer P, Winkelman JW. Restless legs syndrome: demographics, presentation, and differential diagnosis.Geriatrics. 2007;62:26-29.

55. Byrne R, Sinha S, Chaudhuri KR. Restless legs syndrome: diagnosis and review of management options. Neuropsychiatr Dis Treat. 2006;2:155-164.

56. Yoakum R. Night walkers. Do your legs seem to have a life of their own? Your torment has a name. Mod Maturity. $1994 ; 55: 82-84$.

57. Garcia-Borreguero D, Kohnen R, Silber $M H$,et al. The long-term treatment of restless legs syndrome/WillisEkbom disease: evidence-based guidelines and clinical consensus best practice guidance: a report from the International Restless Legs Syndrome Study Group. Sleep Med. 2013;14:675-684.

58. Aurora RN, Kristo DA, Bista SR, et al.The treatment of restless legs syndrome and periodic limb movement disorder in adults-an update for 2012: practice parameters with an evidence-based systematic review and metaanalyses: an American Academy of Sleep Medicine Clinical Practice Guideline. Sleep. 2012;35:1039-1062.

59. Owens RL,Malhotra A. Sleep-disordered breathing and COPD: theoverlap syndrome.Respir Care.2010;55:13331346.

60. Sanders $\mathbf{M H}$, Newman AB, Haggerty $C L$, et al. Sleep and sleep disordered breathing in adults with predominantly mild obstructive airway disease. Am J Respir Crit Care Med. $2003 ; 167: 7-14$

61. Zamarrón C, Garcia Paz V, Morete E, Matías FC. Association of chronic obstructive pulmonary disease and obstructive sleep apnea consequences. Int J COPD. 2008;3:671-682.

62. Pronzato C. Chronic obstructive pulmonary disease and obstructive sleep apnea. Association, consequences and treatment. Monaldi Arch Chest Dis. 2010;73:155-161.

63. Budhiraja R, Kayyali US, Karamsetty $M$, et al. Estrogen modulates xanthine dehydrogenase/xanthine oxidase activity by a receptor-independent mechanism. Antioxid Redox Signal. 2003;5:705-711.
64. Kayyali US, Budhiraja R, Pennella CM, et al. Upregulation of xanthine oxidase by tobacco smoke condensate in pulmonary endothelial cells. Toxicol Appl Pharmacol. $2003 ; 188: 59-68$.

65. Chaouat A, Weitzenblum E, Kessler R, et al. Sleep-related $\mathrm{O}_{2}$ desaturation and daytime pulmonary haemodynamics in COPD patients with mild hypoxaemia. Eur Respir J. $1997 ; 10: 1730-1735$.

66. Hawrylkiewicz I, Sliwinski P, Gorecka D, Plywaczewski R, Zielinski J. Pulmonary haemodynamics in patients with OSAS or an overlap syndrome. Monaldi Arch Chest Dis. 2004;61:148-152.

67. Gan WQ, Man SF, Senthilselvan A, Sin DD. Association between chronic obstructive pulmonary disease and systemic inflammation: a systematic review and a metaanalysis. Thorax. 2004;59:574-580.

68. McNicholas WT. Chronic obstructive pulmonary disease and obstructive sleep apnea overlaps in pathophysiology, systemic inflammation, and cardiovascular disease. Am J Respir Crit Care Med. 2009;180:692-700.

69. Marin JM, Soriano JB, Carrizo SJ, Boldova A, Celli BR. Outcomes in patients with chronic obstructive pulmonary disease and obstructive sleep apnea: the overlap syndrome. Am J Respir Crit Care Med. 2010;182:325-331.

70. Mermigkis C, Kopanakis A, Foldvary-Schaefer N, et al. Health-related quality of life in patients with obstructive sleep apnoea and chronic obstructive pulmonary disease (overlap syndrome). Int J Clin Pract. 2007;61:207-211.

71. Chaouat A, Weitzenblum E, Krieger J, Oswald M, Kessler R. Pulmonary hemodynamics in the obstructive sleep apnea syndrome. Results in 220 consecutive patients. Chest. 1996;109:380-386.

72. Collop NA, Anderson WM, Boehlecke B, et al. Clinical guidelines for the use of unattended portable monitors in the diagnosis of obstructive sleep apnea in adult patients. Portable Monitoring Task Force of the American Academy of Sleep Medicine. J Clin Sleep Med. 2007;3:737-747.

73. de Miguel J, Cabello J, Sánchez-Alarcos JM, Alvarez-Sala $R$, Espinós D, Alvarez-Sala JL. Long-term effects of treatment with nasal continuous positive airway pressure on lung function in patients with overlap syndrome. Sleep Breath. $2002 ; 6: 3-10$.

74. Peker Y, Hedner J, Johansson A, Bende M. Reduced hospitalization with cardiovascular and pulmonary disease in obstructive sleep apnea patients on nasal CPAP treatment. Sleep. 1997;20:645-653.

75. Machado MC, Vollmer WM, Togeiro SM, et al. CPAP and survival in moderate-to-severe obstructive sleep apnoea syndrome and hypoxaemic COPD. Eur Respir J. 2010;35:132-137.

76. Kolodziej MA, Jensen L, Rowe B, Sin D. Systematic review of noninvasive positive pressure ventilation in severe stable COPD. Eur Respir J. 2007;30:293-306. 
77. Berry RB, Budhiraja R, Gottlieb DJ, et al. Rules for scoring respiratory events in sleep: update of the 2007 AASM Manual for the Scoring of Sleep and Associated Events. Deliberations of the Sleep Apnea Definitions Task Force of the American Academy of Sleep Medicine. J Clin Sleep Med. 2012;8:597-619.

78. O'Donoghue FJ, Catcheside PG, Ellis EE, et al. Sleep hypoventilation in hypercapnic chronic obstructive pulmonary disease: prevalence and associated factors. Eur Respir J. $2003 ; 21: 977-984$.

79. Tarrega J, Anton A, Guell R, et al. Predicting nocturnal hypoventilation in hypercapnic chronic obstructive pulmonary disease patients undergoing long-term oxygen therapy. Respiration. 2011 ;82:4-9.

80. Piper AJ. Nocturnal hypoventilation-identifying \& treating syndromes. Indian J Med Res.2010;131:350-365.

81. HolmedahI NH, Øverland B, Fondenes O, Ellingsen I, Hardie JA. Sleep hypoventilation and daytime hypercapnia in stable chronic obstructive pulmonary disease. Int J Chron Obstruct Pulm Dis. 2014:9;265-275.

82. Javaheri S, Shore NS, Rose B, Kazemi H. Compensatory hypoventiltion in metabolic alkalosis. Chest. 1982;81:296301 .

83. Cooper CB, Howard P. An analysis of sequential physiologic changes in hypoxic cor pulmonale during long-term oxygen therapy. Chest. 1991;100:76-80.

84. McEvoy RD, Pierce RJ, Hillman D, et al. Nocturnal noninvasive nasal ventilation in stable hypercapnic COPD: a randomised controlled trial. Thorax. 2009;64:561-566.

85. Angelis GD, Sposato B, Mazzei L, et al. Predictive indexes of nocturnal desaturation in COPD patients not treated with long term oxygen therapy. Eur Rev Med Pharmacol Sci. $2001 ; 5: 173-179$.

86. Roussos C. Function and fatigue of respiratory muscles. Chest. 1985;88:124S-132S.

87. Clinical indications for noninvasive positive pressure ventilation in chronic respiratory failure due to restrictive lung disease, COPD, and nocturnal hypoventilation-a consensus conference report. Chest. 1999;1 16:521-534.

88. Díaz-Lobato S, Alises SM, Rodríguez EP. Current status of noninvasive ventilation in stable COPD patients. Int J Chron Obstruct Pulm Dis. 2006:1;129-135.

89. Ekkernkamp E, Storre JH, Windisch W, Dreher M. Impact of intelligent volume assured pressure support on sleep quality in stable hypercapnic chronic obstructive pulmonary disease patients: a randomized, crossover study. Respiration. 2014;88:270-276.

90. Fletcher EC, Scott D, Qian W, Luckett RA, Miller CC, Goodnight-White S. Evolution of nocturnal oxyhemoglobin desaturation in patients with chronic obstructive pulmonary disease and a daytime PaO2 above 60 torr. Am Rev Respir Dis. $1991 ; 144: 401-425$.

91. Ramsey R, Mehra R, Strohl KP. Variations in physician interpretation of overnight pulse oximetry monitoring. Chest. $2007 ; 132: 852-859$.
92. Zhang J, Wang Y, Feng J, Sun X. Sleep induced hypoxemia in chronic obstructive pulmonary disease.JNMA J Nepa Med Assoc. 2013;52:528-533.

93. Becker HF, Piper AJ, Flynn WE, et al. Breathing during sleep in patients with nocturnal desaturation. Am J Respir Crit Care Med. 1999;159:112-118.

94. Poongkunran C, Budhiraja R. Sleep board review question: nocturnal hypoxemia in COPD. Southwest J Pulm Crit Care. 2013;6:12-14.

95. Fletcher EC, Levin DC. Cardiopulmonary hemodynamics during sleep in subjects with chronic obstructive pulmonary disease. The effect of short- and long-term oxygen. Chest. 1984;85:6-14.

96. Mueller PT, Gomes MD, Viegas CA, Neder JA. Systemic effects of nocturnal hypoxemia in patients with chronic obstructive pulmonary disease without obstructive sleep apnea syndrome. J Bras Pneumol. 2008;34:567-574.

97. Sergi M, Rizzi M, Andreoli A, Pecis M, Bruschi C, Fanfulla F. Are COPD patients with nocturnal REM sleep-related desaturations more prone to developing chronic respiratory failure requiring long-term oxygen therapy? Respiration. 2002;69:117-122

98. Perin C, Fagondes SC, Casarotto FC, Pinotti AF, Menna Barreto SS, Dalcin PdeT. Sleep findings and predictors of sleep desaturation in adult cystic fibrosis patients. Sleep Breath. 2012;16:1041-1048.

99. McNicholas WT, Fitzgerald MX. Nocturnal deaths among patients with chronicbronchitis and emphysema. BMJ (Clin Res Ed). 1984;289:878.

100. Fletcher EC, Donner CF, Midgren B, et al. Survival in COPD patients with a daytime $\mathrm{PaO} 2$ greater than $60 \mathrm{~mm} \mathrm{Hg}$ with and without nocturnal oxyhemoglobin desaturation. Chest. $1992 ; 101: 649-655$

101. O'Reilly P, Bailey W. Long-term continuous oxygen treatment in chronic obstructive pulmonary disease: proper use, benefits and unresolved issues. Curr Opin Pulm Med. 2007;13:120-124

102. Fletcher EC, Luckett RA, Goodnight-White S, Miller CC Qian W, Costarangos-Galarza C. A double-blind trial of nocturnal supplemental oxygen for sleep desaturation in patients with chronic obstructive pulmonary disease and a daytime PaO2 above $60 \mathrm{~mm} \mathrm{Hg}$. Am Rev Respir Dis. 1992;145:1070-1076.

103. Chaouat A, Weitzenblum E, Kessler R, et al. A randomized trial of nocturnal oxygen therapy in chronic obstructive pulmonary disease patients. Eur Respir J. 1999;14:1002 1008.

104. Cranston JM, Crockett AJ, Moss JR, Alpers JH. Domiciliary oxygen for chronic obstructive pulmonary disease. Cochrane Database Syst Rev. 2005:CD001744.

105. Krachman SL, Chatila W, Martin UJ, et al. Effects of lung volume reduction surgery on sleep quality and nocturnal gas exchange in patients with severe emphysema. Chest. $2005 ; 128: 3221-3228$. 